\title{
Lung cancer investigations and treatment Greater possibilities to improve patients' quality of life
}

Lung cancer has for many years been a disease with poor prognosis and still is. However, new techniques for diagnostic procedures and the introduction of new treatment alternatives give hope that the near future will bring maybe not cure for all patients but the possibility to turn the disease from a killer with a short life expectancy to a disease of chronic nature for which various treatment options keep the disease stable and gives the patient a good quality of life.

The introduction of positron emission tomography-computed tomography (PETCT) as a part of lung cancer examinations has given us the possibility of creating a map of each patient early in the process of diagnostic procedures. However, it is important to state that PET-CT can not be used alone to decide what stage of disease the patient has and thereby design a treatment programme. PET-CT needs to be combined with other diagnostic procedures, such as bronchoscopy, endobronchial ultrasound (EBUS), endoscopic ultrasound (EUS) and mediastinoscopy, in order to have complete information about where the lung cancer is located. EBUS and EUS have proven to be able to replace mediastinoscopy in many situations in order to examine lymph nodules in mediastinum. Correct staging of mediastinum has historically been the most difficult part in lung cancer examinations to obtain a correct TNM (tumour, node, metastases) classification. With the use of PET-CT, EBUS, EUS, mediastinoscopy and, in some instances, video assisted thoracoscopy
(VATS), we now have the techniques to be able to obtain a correct TNM classification to base our treatment on.

Small lung tumours have always been difficult to obtain cytological or histological tissue from, but with the introduction of electromagnetic navigation systems and EBUS it is nowdays often possible to obtain tissue from lesions $1-2 \mathrm{~cm}$ in size even if they are located in the peripheral lung.

Bronchoscopy in combination with autofluorescence is a new technique that gives the possibility to visualise and sample small cancer lesions in the bronchial mucosa before they are visible in radiographic examinations. This technique can therefore be used in screening. It can also be of great value in pre- and postoperative settings.

For curable lung cancer treatment, surgery is still the main alternative. Surgery is often combined with other treatment options, such as chemotherapy and radiotherapy. Postoperative adjuvant chemotherapy is today considered standard in stage-II and -IIIA disease. More and more information also supports that neoadjuvant chemotherapy has a place in selected cases in order to be able to perform radical surgery after pre-treatment with cytostatic drugs. In breast cancer, multimodal treatment is the choice for many patients and lung cancer treatment is also moving in that direction.

Patients who are not suitable for surgery can sometimes have the alternative to be treated with stereotactic radiotherapy as a curable treatment option.
R. Öhman

Department of Pulmonary Medicine, University Hospital of Skåne, Lund, Sweden

\section{Correspondence}

R. Öhman

Clinical Sciences

Respiratory Diseases and Allergology

SUS/Lund

Lund

22185

Sweden

ronny.ohman@skane.se 
The majority of patients have at the time of diagnosis stage-IIIB or -IV disease which is considered incurable. Cytostatic chemotherapy has been the treatment option for this group. Also, for some patients, radiotherapy concomitantly or in sequence can be of value. Radiosensitising agents are now being tested in order to improve the effect of the radiotherapy.

Maintenance treatment with cytostatic drugs has been tried historically but the side effects have been difficult to handle. However, there is now information that Pemetrexed can be used as maintenance treatment in order to keep the lung cancer in a stable state. Also Erlotinib have been tried as maintenance therapy with some positive results.

Other target therapy substances besides Erlotinib/gefinitib are tested and hopefully they will be on the market shortly.

Bone metastasis is usually painful and also worsening the prognosis. Besides radiotherapy which is usually first option for treatment, bisphosphonates, such as zoledronic acid may give treatment benefits for disease control.

Endobronchial intervention in order to stop tumour from blocking the air in trachea and main bronchi is possible by the use of laser or electrocautery in combination with rigid bronchoscopy. By placing stents, it is also possible to prolong the result. Such intervention is usually only performed in major lung cancer centres and it is, therefore, important to identify suitable patients in time so that they can be referred to these treatment centres in order to benefit from this option.

Very exiting studies are ongoing to investigate the possibility for immune therapy. Lung cancer vaccines are in phase three studies and hopefully it can be shown to give impact on survival.

So the future for lung cancer patients will change and the treatment options will be better making lung cancer a disease possible to live with.

In this issue of Breathe, we have some interesting articles on lung cancer covering the aforementioned topics. W. De Wever discusses the use of imaging techniques in lung cancer, including computed tomography, magnetic resonance imagery and 3-dimensional radiology, F. J. F. Herth discusses bronchoscopic techniques while R. Rami Porta et al. discuss the introduction of the 7th TNM classification system. 